\title{
Absence of Quantitative Improvement in Neuropsychological Profiles in Patients Who Exhibit Moderate Brain Impairment: Comparisons of Cross-Sectional and Longitudinal Data (1 through 4 Years Post-Injury)
}

\author{
Sandra G. Tiller, Linda S. St-Pierre, Michael A. Persinger \\ Clinical Neuropsychology Laboratory, Department of Psychology and Behavioural Neuroscience Program, \\ Laurentian University, Sudbury, Canada \\ Email: mpersinger@laurentian.ca
}

Received January 15, 2013; revised March 25, 2013; accepted May 8, 2013

Copyright (C) 2013 Sandra G. Tiller et al. This is an open access article distributed under the Creative Commons Attribution License, which permits unrestricted use, distribution, and reproduction in any medium, provided the original work is properly cited.

\begin{abstract}
A total of 127 adult patients who had sustained an impact of significant mechanical energy to their skulls during motor vehicle incidents were given thorough neuropsychological, cognitive and personality assessments between 0.5 years and 4 years after the event. Cross-sectional analysis indicated no statistically significant objective changes in patients as a function of yearly intervals. However there was strong evidence of significant deterioration of neuropsychological proficiency and efficiency between 0.3 to 1.0 years after the injury. A subset $(n=20)$ of patients who displayed moderately severe neuropsychological impairment when assessed about 1 year after the injury showed no statistically significant changes when reassessed about 1.5 years later (2.5 years after the brain trauma). These results challenge the traditional concept of "recovery" following a traumatic brain injury and indicate that insidious processes that adversely affect neurocognitive capacity may emerge 0.5 years after the trauma. Post-hoc analysis indicated that the occurrence of unconsciousness or its duration at the time of the injury minimally affected the magnitude of subsequent indices of neuropsychological impairment but influenced the incidence of electroencephalographic theta activity during the years following the injury.
\end{abstract}

Keywords: TBI; Neuropsychological Impairment; Test-Retest Reliability; Personality; Electroencephalographic Indicators

\section{Introduction}

During the months to years following a traumatic brain event (closed head injury) many patients exhibit significant and self-evident changes in their neurocognitive capacities [1-3]. According to the Neuropsychologica Principia Brevita [1] contemporary medical and legal approaches to the actual neurophysical changes associated with the transfer of mechanical energy through the cerebral volume do not reflect the principles of neuroscience or the temporal complexity of human performance. Legal definitions, political agendas, or consensus of professionals that comprise regulatory bodies and colleges do not represent the scientific method and often obscure, discredit, or ignore the quantitative data that contradict the presumptions of the group.
The time required for the changes in standardized, norm-referenced performance based measurements of neuropsychological function to asymptote exhibits significant individual variability that is only weakly to moderately correlated with traditional variables such as scores for the Glasgow Coma Scale (GCS), the duration of coma, or the interval of posttraumatic amnesia. For patients who have sustained mild to moderate brain traumas, that are often categorized as concussions or contusions [4], the traditional predictors of outcome are not valid [5]. Definitions of "mild traumatic brain injury", such as: 1) cranial trauma, 2) loss of consciousness for $20 \mathrm{~min}$ or less, 3) GCS scores between 13 and 15 at the time of admission, 4) posttraumatic dysmnesia of less than $24 \mathrm{hr}$ or 5) absence of CT-discernable anomalies are arbitrary and 
sometimes contrived criteria that ignore the physics of sudden displacements or impacts of mechanical energy within brain space [6-8].

Estimates for the duration required for neurocognitive capacities to asymptote after brain trauma have been derived primarily from patients who have sustained very severe brain injuries due to mechanical impact or cerebral vascular accident (CVA), i.e., stroke, and have often employed very crude inferences, such as average intelligence scores [9] to estimate the asymptote for "recovery". Estimates for patients who have sustained mild to moderate brain traumas are implicitly assumed to be linear extrapolations from the data of more severely impaired cases. However the effect may be non-linear. There is now evidence that mild brain trauma may result in more severe and numerous problems with adaptation because these patients have the cognitive capacity to appreciate and to remember the changes which emerged after the injuries.

Litigious processes often select two years following the day of the brain injury or trauma as a reference by which long-term or permanent dysfunction can be inferred. Considering the marked individual variability in normal neurocognitive capacity and the large proportion of variance due to unknown quantities, e.g., direction of impact or the focal or distributed forces through brain space, this two year limit may not have empirical support. The present study was designed to determine if there are any statistically significant changes in a variety of traditional and non-traditional domains of neuropsychological tests during the first four years following a brain trauma.

To answer this question, both a cross-sectional design and longitudinal (repeated measure) design were employed. We reasoned that with our sample size any statistically significant change of a test score between cohorts would also be evident within a smaller sample of patients who were tested twice (employed as their own controls) because of the reduction in individual variability. We also selected a broad range of measures that included standardized scores for personality, intelligence, educational achievement, and both the traditional indices and newer indices, such as the dichotic word listening test, of blatant and subtle alterations in neurocognitive capacities. Objective measurements rather than either the subjective experiences of the patient or caregiver were emphasized because we have found that patients often habituate to their impairments and, as a result, report they are "normal" when objectively their deficits have not changed quantitatively.

\section{Methods}

\subsection{Data Base}

\subsubsection{Cross-Sectional Study: Within 4 Years of Injury}

A total of 100 patients who had been referred successively for a complete neuropsychological assessment over a 5-year period (1990 through 1994) were included as subjects. More than $80 \%$ of the cases were referred by rehabilitation companies while most of the remaining cases had been referred directly by lawyers. There were 55 men and 45 woman, whose mean age was $34.3(\mathrm{SD}=12.9)$ years old ( $95 \%$ were between 16 years and 55 years old). Twelve percent $(12 \%)$ of the population preferred their left hands and $14 \%$ preferred their left feet. The subjects were aggregated in groups according to the time since the injury: less than 1 year, 1 through 2 years, 2 through 3 years and more than 3 years $(90 \%<4$ years $)$. The mean and standard deviation for the numbers of years of formal education were 11.7 and 2.8 , respectively, while the durations in days of posttraumatic amnesia were 20 and 30 , respectively. There was no significant difference (chisquared $<1.00$ ) in the latency of testing after the injury with respect to those who had been unconscious for at least $5 \mathrm{~min}(53 \%)$ or had remained relatively lucid according to both subjective report and the data from the medical records.

\subsubsection{Longitudinal Study}

A total of 20 patients (10 men, 10 women) from the crosssectional study (mean and SD for age $=33$ years, 7 years, respectively) were given two, separate complete neuropsychological assessments. The first assessment occurred between 0.5 and 1.5 years (mean $=1.0$ year) after the brain trauma (all associated with motor vehicle incidents) and the second assessment was given between 1 year and 2.5 years later (average time of second assessment $=1.5$ years after the first assessment). The ages, formal education, proportion who reported unconsciousness after the injury, duration of PTA and handedness did not differ significantly from the major $(\mathrm{n}=100)$ population.

\subsubsection{Cross-Sectional Study: Within One Year of Injury}

A total of 62 patients (mean age $=31$ years; $S D=10$ years) were included in this analysis; $57 \%$ were men while $42 \%$ were women. This sample was the aggregate of 35 patients from the first study who had sustained brain trauma less than one year before the assessment (years 1990 through 1993) and an additional 27 patients who had been assessed during the years 1994 and 1995. This group did not differ (chi-squares < 2.00) from the first sample with respect to proportion of sinistrals, occurrence of unconsciousness, or gender. These 27 patients were incorporated into the study because their assessment times were assigned due to the policy of referral within a few months after a brain trauma and involved minimal screening or selection bias.

To facilitate comparisons, only the $\mathrm{z}$ scores for each of the measurements were employed. The means and standard deviations from which the $\mathrm{z}$ scores were derived 
was based upon the 50, age matched, normal individuals (within the data base) who had been assessed for other reasons. They did not differ from a variety of studies $(n=$ 150) that have employed 20 to 30 year old university students as the sample populations.

\subsection{General Procedures}

The clinical neuropsychological assessment usually required 1.5 to 2 days $(7 \mathrm{hr} /$ day). The testing also included a brief (30 min) structured neuropsychological interview, assessment of neurological indicators [3] and a one-hour screening electroencephalographic measurement $(\mathrm{O} 1, \mathrm{~T} 3$, $\mathrm{F} 7, \mathrm{O} 2, \mathrm{~T} 4, \mathrm{~F} 8)$ in order to discern electrical seizure activity and cardiac aperiodicity and to help substantiate the validity of psychometric testing. The structured interview included data such as types of medication, changes in relationships within the family, and alterations in libido.

Measurements described in greater detail elsewhere [10] were taken for the following procedures: Wechsler Adult Intelligence Scales (WAIS-R), Verbal and Performance scores (standardized), Wechsler Memory Scale (standardized), means of the spelling, reading and arithmetic sections of the Wide Range Achievement Test (WRAT-2), Finger Taps for the right and left index fingers $(\mathrm{n} / 10 \mathrm{sec})$, Foot Tap for the right and left feet $(\mathrm{n} / 10$ $\mathrm{sec}$ ), Trails A (sec), Trails B (sec), Category Test (total errors), Design Fluency, numbers of blocks to criterion for the Conditioned Spatial Association Test [11], Spatial Span, Rey-Ostereith (RO) Copy, Rey-Ostereith Memory (+30 min), Tactual Performance Test (TPT) for the dominant (D) and non-dominant (ND) hand, TPT (memory) TPT (localization), Speech Sounds (correct, first 30 trials), Seashore Rhythms Test (number correct), Auditory Closure (number correct), Sentence Memory (number correct), Digit Span (standardized score) from the WAIS-R, FAS (Verbal Fluency), Dichotic Word Listening for the right and left ears, color word score (T) from the Stroop, Finger Gnosis (errors for the right and the left hand), Finger Graphaesthesia, (errors for the right and the left hand), Toe Gnosis (errors for the right and the left foot) and Toe Graphaesthesia (errors for right and the left foot). The MMPI-168 [12] was also administered as well as Roberts' inventory of Complex Partial Epileptic-Like Signs $[13,14]$.

For the electroencephalographic screening, the subject sat in a comfortable arm chair that was housed within a commercial acoustic chamber. During the first $40 \mathrm{~min}$, bipolar measurements between O1,O2, T3,T4 and F7,F8 were recorded while during the last $20 \mathrm{~min}$ comparisons between the left and the right hemispheres for the logical combinations of montages: T-F, T-O and F-O, were completed for each hemisphere. Heart rate was recorded. All measurements employed a Grass P79 unit.
The proportion of alpha rhythms (defined as the synchronized activity between 8 and $13 \mathrm{~Hz}$ ) was computed for the first $5 \mathrm{~min}$ of the measurement by estimating the proportion of each $20 \mathrm{sec}$ section of the record that contained alpha rhythms $(0 \%$ to $100 \%)$ and then calculating the average for those 15 estimates. This was completed for the bipolar occipital, temporal and frontal measurements. Mean heart rate (beats/min) was taken by totalling the numbers of QRSs for 3 separate, $30 \mathrm{sec}$ sections of the record during the last $5 \mathrm{~min}$ of the session. The latencies, in min, before alpha dropout from the occipital regions (defined as the conspicuous attenuation of alpha rhythms) and the latency before spindles (fast, synchronous bursts of $13-15 \mathrm{~Hz}$ with durations of about $1 \mathrm{sec}$ ) occurred (primarily) first in the prefrontal region, were recorded.

Nominal measures (presence, absence) were completed for theta bursts during bipolar measurements over the occipital, temporal and frontal lobes. Qualitative anomalies, specifically slow-wave bursts and atypical spikes that emerged from the intrahemispheric comparisons (e.g., right frontooccipital vs left frontooccipital) were also recorded for each of the $5 \mathrm{~min}$ to $7 \mathrm{~min}$ samplings for these montages. A global anomaly score for the general record, ranging from 0 (normal, referenced to approximately 500 volunteers that have been tested in the chamber during the previous 20 years) to 3 (maximum strangeness). The anomaly score was due to the presence of: theta bursts while awake, odd, spiky activity over the temporal lobes, fast beta over the temporal lobes, subvigal beta activity (primarily prefrontal), odd paroxysmal activity, and marked asymmetries between two homologous hemispheric measurements. The occurrence of a classic epileptiform sequence during the measurement was automatically given a score of 3 . The criteria were derived from Niedermeyer and da Silva [15] and the 40 years of experience of the third author.

\subsection{Statistical Analyses}

All analysis involved SPSS software on a Vax 4000 computer. For the cross-sectional data, one-way analyses of variance (ONEWAY) were completed for each of the dependent measures as a function of the time since the brain trauma. Post hoc analysis employed Scheffe's tests set at $\mathrm{p}<0.05$. Chi-squared analyses were completed for relevant nominal, e.g., unconsciousness vs consciousness immediately after the injury, and ordinal scales. For the longitudinal (test-retest) cases, paired t-tests were completed for the same dependent measures. The criteria for acceptable statistical significance was $\mathrm{p}<0.01$. For the third study which investigated potential non-linear changes over time during the first year (only) after the injury, the weighted (for unequal n sizes/temporal increment) linear, 
quadratic and cubic terms (POLYNOMIAL) were extracted.

\section{Results}

\subsection{Major Cross-Sectional Study}

The means and standard deviations for each of the neuropsychological and cognitive measures as a function of the time since the injury are shown in Table 1 while the indices of personality (MMPI scales) are shown in Table 2. None of the oneway analyses of variance demonstrated any statistically significant differences between any of the measures as a function of the time since the injury. There were also no statistically significant differences for the various groups for the $\mathrm{T}$-scores for the various scales from the personality inventory.

Although the standardized global impairment scores

Table 1. Means and standard deviations for cognitive (standardized) and neuropsychological (raw) scores for patients who were tested within one year ( $<1$ yr), 1 to 2 years, 2 to 3 years or 3 or more years after a traumatic brain injury.

\begin{tabular}{|c|c|c|c|c|c|c|c|c|}
\hline \multirow{2}{*}{ Scale } & \multicolumn{6}{|c|}{ Time since injury } & & \\
\hline & \multicolumn{2}{|c|}{$<1 \mathrm{yr}$} & \multicolumn{2}{|c|}{2 yrs } & \multicolumn{2}{|c|}{3 yrs } & \multicolumn{2}{|c|}{$>3$ yrs } \\
\hline Verbal IQ & 87 & 11 & 89 & 13 & 91 & 10 & 87 & 12 \\
\hline Perform IQ & 89 & 11 & 94 & 13 & 98 & 14 & 92 & 18 \\
\hline Achievement & 89 & 14 & 91 & 12 & 91 & 12 & 93 & 13 \\
\hline Digit span & 8.2 & 2.8 & 8.9 & 2.4 & 8.6 & 3.1 & 9.1 & 3.1 \\
\hline Z-Impair & -1.5 & 1 & -1.3 & 1.4 & -1.2 & 1.1 & -1.6 & 1.6 \\
\hline \multicolumn{9}{|c|}{ Raw scores } \\
\hline H-R index & 0.55 & 0.29 & 0.33 & 0.26 & 0.44 & 0.23 & 0.39 & 0.32 \\
\hline Fing Tap L & 43.4 & 9.2 & 43.9 & 8.9 & 42.3 & 10.4 & 42.8 & 12 \\
\hline Foot Tap R & 37.8 & 10.6 & 36.5 & 11.2 & 34.8 & 12.6 & 41.7 & 14.1 \\
\hline Foot Tap L & 33.4 & 11.4 & 36.8 & 11.3 & 34.4 & 13.1 & 36.4 & 13 \\
\hline Trails A & 44.4 & 21.8 & 34.8 & 15.3 & 42.8 & 25 & 41.1 & 22 \\
\hline Trails B & 115 & 81.1 & 79.5 & 27.7 & 103.3 & 75.2 & 114.4 & 113.8 \\
\hline Category & 70.7 & 24.5 & 61.7 & 27.9 & 64.4 & 34.8 & 60.5 & 39 \\
\hline Design F. & 20.8 & 11.4 & 17.7 & 9.6 & 18.8 & 7.7 & 18.2 & 10.3 \\
\hline CSAT & 70.4 & 55.5 & 73.2 & 67.2 & 60.1 & 55.2 & 66.1 & 68.8 \\
\hline Spatial Sp & 5.3 & 1.1 & 5.7 & 1.2 & 5.4 & 0.7 & 4.2 & 1.6 \\
\hline RO-copy & 29.5 & 3.7 & 30 & 2.2 & 27.8 & 4.3 & 29.6 & 4.9 \\
\hline RO-mem & 14.4 & 6.7 & 17.5 & 5.6 & 14.6 & 7 & 14.6 & 7.4 \\
\hline TPT-D & 6.7 & 3.3 & 5.1 & 1.5 & 6.2 & 2.4 & 6.6 & 3.1 \\
\hline TPT-ND & 5.2 & 2.7 & 5 & 2.7 & 4.9 & 2.5 & 6.5 & 3.3 \\
\hline TPT-MEM & 6.3 & 1.7 & 7.2 & 1.5 & 6.9 & 1.6 & 7.1 & 2.1 \\
\hline Seashore & 23.6 & 2.5 & 26.4 & 3 & 23.4 & 4.6 & 24.4 & 7.5 \\
\hline Aud Clos & 15.1 & 6 & 15.8 & 6.7 & 16.2 & 5.3 & 13.6 & 6.3 \\
\hline Sent Mem. & 15.9 & 2.8 & 16 & 2.9 & 16.3 & 3.2 & 13.9 & 4.3 \\
\hline Verbal Fl. & 31.4 & 11.4 & 40.3 & 12 & 37.7 & 12.5 & 36.6 & 14.4 \\
\hline DICH-LE & 34.7 & 7.5 & 34.9 & 9.7 & 37.5 & 6.2 & 33.2 & 14.6 \\
\hline DICH-RE & 37.8 & 10.3 & 33.6 & 12 & 35.9 & 11.4 & 33.6 & 13.7 \\
\hline Stroop CW & 39.6 & 8.8 & 43.1 & 9.3 & 42 & 10.2 & 38.8 & 13.3 \\
\hline Fing Agn R & 1.1 & 1.7 & 0.7 & 1.6 & 1.2 & 2.3 & 1.4 & 2.6 \\
\hline Fing Agn L & 1.1 & 1.7 & 0.6 & 1.3 & 1.2 & 1.3 & 1.6 & 3.5 \\
\hline Fing Graph R & 3 & 2.6 & 2.6 & 2.1 & 2.1 & 2.9 & 3.5 & 4.5 \\
\hline Fing Graph L & 2.5 & 2.5 & 1.3 & 1.2 & 1.9 & 3.2 & 4.3 & 5 \\
\hline Toe Agn R & 3.5 & 2.1 & 4.9 & 2.3 & 5.1 & 3.2 & 4.7 & 3.6 \\
\hline Toe Agn L & 4.7 & 2.7 & 3.9 & 2.1 & 4.3 & 2.7 & 4.9 & 2.2 \\
\hline Toe Graph R & 6.7 & 4.2 & 6.3 & 3.3 & 5.7 & 4.6 & 9.5 & 5.2 \\
\hline Toe Graph L & 6.4 & 3.5 & 5.5 & 3.6 & 5.7 & 3.6 & 9.2 & 5.4 \\
\hline CPES-signs & 60.6 & 43.7 & 68 & 42.2 & 48.5 & 26.6 & 87.3 & 27.1 \\
\hline
\end{tabular}


derived from 31 measures and the H-R Impairment Indices were, on average, suggestive of mild to moderate deficits, these values do not necessarily reflect discrepancies within the individual patient. The "diagnosis" or hypothesis that a recent brain impairment has occurred usually requires a significant discrepancy (deficit) between the patient's standardized scores for intelligence and the standardized scores for neuropsychological criteria [16]. We calculated the difference between the mean of the standardized scores for verbal intelligence, performance intelligence and global memory (WMS) and subtracted it from the global neuropsychological score. The mean and SD were -0.8 and 0.9 , respectively. Twentysix percent $(26 \%)$ of all of the patients showed neuropsychological scores that were between -1.1 and -1.9 standard deviations below their cognitive scores while an additional $8 \%$ displayed deficits that were more than -2.00 standard deviations from these scores. Chisquared analysis $(\mathrm{df}(4)=2.07)$ indicated no significant difference in the distribution of the $34 \%$ of the population between the different periods of assessment.

The results of the quantitative and qualitative electroencephalographic measures for the cross-sectional study were more revealing (Table 3 ). There was a significant change in the proportion of alpha rhythms over the occipital lobes $[\mathrm{F}(3,96)=4.41, \mathrm{p}<0.01$; eta $=0.41]$ but not the frontal or temporal lobes as a function of time since the brain trauma. Post hoc analysis indicated that the major source of this difference was the lower proportions of occipital alpha rhythms (alpha seconds/min) for the group assessed within 1 year of the brain trauma compared to those assessed two or three years later. The variability of the proportion of alpha rhythms between groups (heterogeneity of variance) was statistically significant over the frontal lobes (Bartlett-Box $F=9.31, \mathrm{p}<0.001$ ) and over the temporal (Bartlett-Box $F=5.70, p<0.01$ ) lobes.

Table 2. Means and standard deviations for standardized (T) scores for the MMPI scales as a function of time in years since the traumatic brain injury ( $\mathrm{n}=20$ to 25 /increment).

\begin{tabular}{|c|c|c|c|c|c|c|c|c|}
\hline \multicolumn{9}{|c|}{ Time (in years) since injury } \\
\hline \multirow{2}{*}{ MMPI scale } & \multicolumn{2}{|c|}{$<1 \mathrm{yr}$} & \multicolumn{2}{|c|}{$1-2$ yrs } & \multicolumn{2}{|c|}{$2-3$ yrs } & \multicolumn{2}{|c|}{$>3$ yrs } \\
\hline & $\mathbf{M}$ & SD & $\mathbf{M}$ & SD & $\mathbf{M}$ & SD & $\mathbf{M}$ & SD \\
\hline $\mathrm{L}$ & 52 & 11 & 54 & 8 & 54 & 8 & 54 & 10 \\
\hline $\mathrm{F}$ & 69 & 13 & 71 & 15 & 68 & 12 & 68 & 13 \\
\hline $\mathrm{K}$ & 48 & 10 & 49 & 8 & 50 & 9 & 47 & 8 \\
\hline Hs & 70 & 14 & 72 & 15 & 74 & 13 & 66 & 16 \\
\hline $\mathrm{D}$ & 73 & 12 & 76 & 18 & 81 & 11 & 74 & 15 \\
\hline Hy & 66 & 12 & 76 & 18 & 81 & 11 & 74 & 15 \\
\hline $\mathrm{Pd}$ & 68 & 12 & 69 & 15 & 68 & 14 & 68 & 12 \\
\hline $\mathrm{Mf}$ & 51 & 13 & 59 & 16 & 55 & 11 & 55 & 15 \\
\hline $\mathrm{Pa}$ & 58 & 13 & 61 & 10 & 64 & 8 & 62 & 14 \\
\hline $\mathrm{Pt}$ & 70 & 17 & 70 & 19 & 74 & 12 & 70 & 18 \\
\hline $\mathrm{Sc}$ & 59 & 11 & 59 & 11 & 61 & 11 & 59 & 12 \\
\hline $\mathrm{Ma}$ & 70 & 11 & 66 & 14 & 66 & 11 & 59 & 12 \\
\hline $\mathrm{Si}$ & 58 & 19 & 58 & 12 & 58 & 12 & 60 & 12 \\
\hline
\end{tabular}

Table 3. Means and standard deviations for various electroencephalographic measures as a function of time since the brain injury.

\begin{tabular}{|c|c|c|c|c|c|c|c|c|}
\hline \multirow{2}{*}{ Variable } & \multicolumn{2}{|c|}{$<1 \mathrm{yr}$} & \multicolumn{2}{|c|}{1 - 2 yrs } & \multicolumn{2}{|c|}{2 - 3 yrs } & \multicolumn{2}{|c|}{$>3$ yrs } \\
\hline & $\mathbf{M}$ & SD & $\mathbf{M}$ & SD & $\mathbf{M}$ & SD & $\mathbf{M}$ & SD \\
\hline \multicolumn{9}{|l|}{ \% alpha } \\
\hline Occipital & 18 & $21 \mathrm{a}$ & 37 & $25 b$ & 40 & $29 b$ & 24 & 21 \\
\hline Temporal & 8 & 17 & 14 & 21 & 8 & 14 & 5 & 7 \\
\hline Frontal & 2 & 15 & 12 & 32 & 11 & 32 & 21 & 41 \\
\hline Alpha dropout (min) & 15.6 & 9.6 & 16.7 & 8.9 & 13 & 8.7 & 17.8 & 9.2 \\
\hline Spindles (min) & 17.7 & 8.9 & 20.3 & 6.7 & 16 & 8.3 & 19.2 & 7.6 \\
\hline Anomalous EEG & 1.4 & $0.9 \mathrm{a}$ & 1.2 & 0.8 & 0.8 & $0.8 \mathrm{~b}$ & 1.5 & 0.9 \\
\hline QRS/sec & 69 & 10 & 69 & 11 & 65 & 11 & 68 & 10 \\
\hline Seizure trains $\%$ & \multicolumn{2}{|c|}{$0 \%$} & \multicolumn{2}{|c|}{$0 \%$} & \multicolumn{2}{|c|}{$0 \%$} & \multicolumn{2}{|c|}{$12 \%$} \\
\hline
\end{tabular}

*a vs $b, \mathrm{p}<0.05$. 
There were no statistically significant differences between time since the injury and the latency (in min) for alpha dropout $[\mathrm{F}<1.00]$ or the sudden appearance (in min) of spindles $[F<1.00]$ that usually began over the prefrontal regions.

The numbers of anomalous characteristics ("spiky" patterns over the frontal lobes, fast beta activity over the prefrontal or temporal lobes, occasional theta bursts) were more frequent within the first year after the brain trauma relative to the longer periods. However the incidence of actual epileptiform, classic seizure trains (paroxysmal spike and slow waves within 3 to $4 \mathrm{~Hz}$ or sharp theta trains while awake) was significantly greater in the group that was referred more than 3 years after the trauma. There were no significant group differences for the average heart rate as defined by numbers of QRS/min.

The proportion of patients who were employing various medications to reduce the problems attributed to their brain traumas are shown in Table 4 . There was a marginal but statistically significant increase in the consumption of antidepressants more than 1 year after the injury relative to within the first year. The proportion of patients who remained married (54\% of the population had been separated/divorced since the incident) or who exhibited post-injury epileptic seizures since the injury did not differ significantly over time (Table 5). Analysis of the ranks of decrease (0) no change (1) or increase (2) for the feeling of closeness to the family (i.e., love) and libido (desire for sexual activity) showed no significant differences $(\mathrm{F}<1.00)$ as a function of time since the brain trauma.

Table 4. Proportion of patients who reported various pharmacological or demographic characteristics as a function of time since the brain trauma.

\begin{tabular}{|c|c|c|c|c|c|c|c|c|c|}
\hline \multicolumn{10}{|c|}{ Time (in years) since injury } \\
\hline Variables & \multicolumn{2}{|c|}{$<1 \mathrm{yr}$} & \multicolumn{2}{|c|}{1 - 2 yrs } & \multicolumn{2}{|c|}{$2-3$ yrs } & \multicolumn{2}{|c|}{$>3 \mathrm{yrs}$} & Total \\
\hline \multicolumn{10}{|l|}{ Drugs } \\
\hline Anticonvulsants & \multicolumn{2}{|c|}{$15 \%$} & \multicolumn{2}{|c|}{$24 \%$} & \multicolumn{2}{|c|}{$11 \%$} & \multicolumn{2}{|c|}{$23 \%$} & $18 \%$ \\
\hline Antidepressants & \multicolumn{2}{|c|}{$8 \%$} & \multicolumn{2}{|c|}{$28 \%$} & \multicolumn{2}{|c|}{$11 \%$} & \multicolumn{2}{|c|}{$33 \%$} & $17 \%$ \\
\hline Analgesics & \multicolumn{2}{|c|}{$33 \%$} & \multicolumn{2}{|c|}{$36 \%$} & \multicolumn{2}{|c|}{$44 \%$} & \multicolumn{2}{|c|}{$42 \%$} & $37 \%$ \\
\hline Anxiolytics & \multicolumn{2}{|c|}{$10 \%$} & \multicolumn{2}{|c|}{$5 \%$} & \multicolumn{2}{|c|}{$6 \%$} & \multicolumn{2}{|c|}{$17 \%$} & $9 \%$ \\
\hline Total Meds & $\mathbf{M}$ & SD & $\mathbf{M}$ & SD & $\mathbf{M}$ & SD & $\mathbf{M}$ & SD & \\
\hline (Range 0 - 7) & 1 & 1.1 & 1.3 & 1.1 & 1.2 & 1.4 & 1.7 & 2 & \\
\hline
\end{tabular}

Table 5. Proportions of patients reporting significant changes in life style or motivation as a function of time in years since the injury.

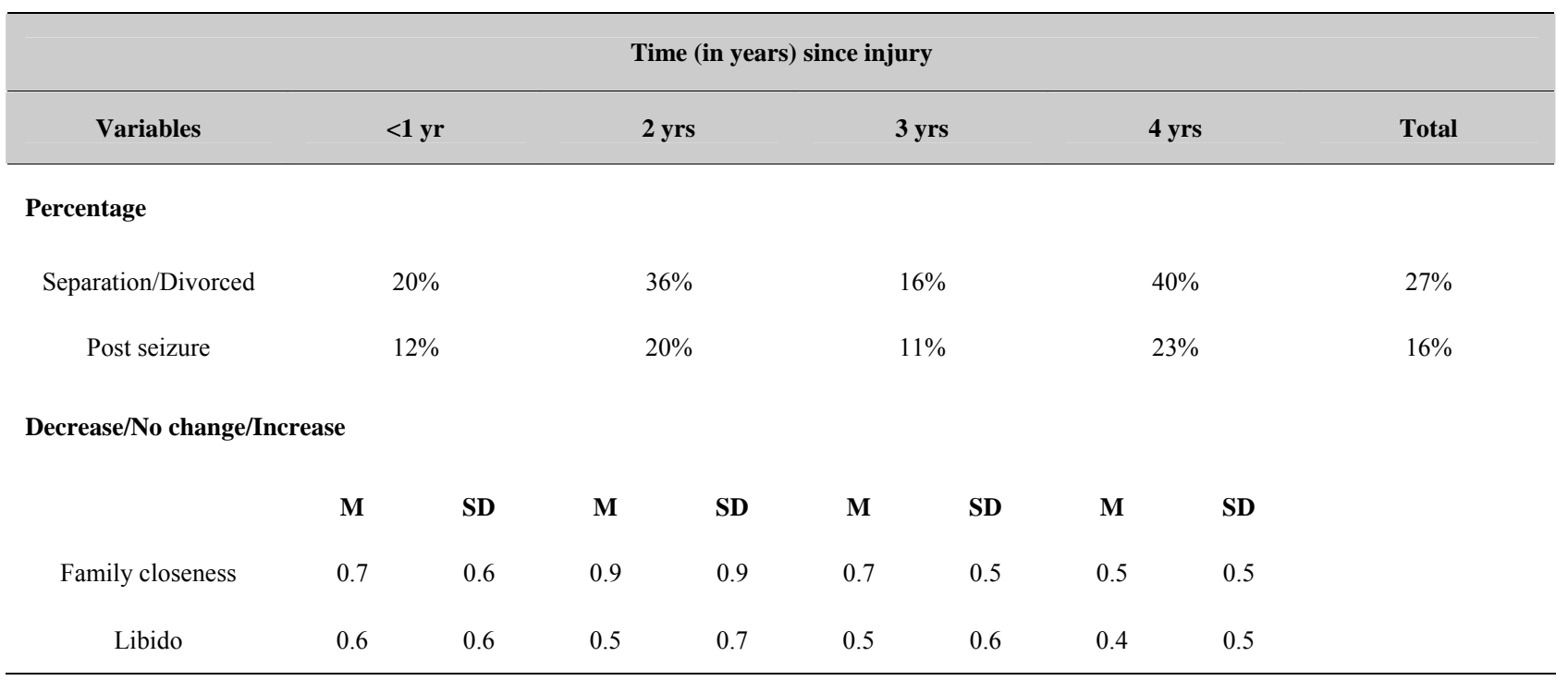




\subsection{Longitudinal Test-Retest Study}

The means and standard deviations for the scores for neuropsychological tests for the first and second measurements for the 20 subjects are shown in Table 6. The t-tests values for differences between the means for the first and second assessments as well as the correlations (r) between the scores for the pairs of measurements (separated by about 1.5 years) are also shown in Table 6.

There were no statistically significant $(p<0.01)$ changes for the major cognitive (inferences of intelligence) measurements or indicators of general impairment (either the mean z-scores for 31 different performance-based tests or the H-R Index) between the first and second assessments. The only statistically significant $(p<0.01)$ improvements between the first and second assessment occurred for the delayed memory component of the Rey-Ostereith Figure $[\mathrm{F}(1,19)=9.08, \mathrm{p}=0.007$; eta-squared $=34 \%)$. If $\mathrm{p}<$ 0.05 were considered statistically significant rather than our a priori criteria of $p<0.01$, then there were also significant improvements (reduction of errors) for scores for the Conditioned Spatial Association Test and (increased correct responses) for the Auditory Closure test. The tests that displayed the strongest test-retest correlations (i.e., $\mathrm{r}$ $>0.70$ ) were the mean Halstead-Reitan Impairment Index, Verbal Intelligence, Performance Intelligence, average Educational Achievement, Complex Partial EpilepticLike Signs, Auditory Closure, Verbal Fluency, Dichotic Word Listening Accuracy for the Right Ear, TPT latency for the non-dominant hand, all of the agility scores for the feet and fingers, and only the errors for toe graphaesthesia but not finger graphaesthesia.

Test-retest analyses for the scales from the MMPI for these 20 patients demonstrated no statistically significant differences over time. There was a weak decrease $(t=$ $2.52, p=0.02)$ in the scaled scores for psychasthenia $(\mathrm{Pt})$ between the first (mean, SD $=69,15$ ) and the second (mean, $\mathrm{SD}=64,15$ ) assessment, respectively, which corresponded to an improvement of about 0.5 standard deviations towards the normative mean. The strongest testretest correlations $(r>0.65 . p<0.001)$ occurred for the following scales: $\mathrm{L}, \mathrm{Hs}, \mathrm{Hy}, \mathrm{Pt}$, and $\mathrm{Si}$. For the other scales, all but the two $\mathrm{K}$ scales were correlated significantly $(\mathrm{p}<0.01)$.

In order to control for possible artifacts as a function of the latency between the injury and the first assessment and between the first and second assessments, partial correlations were completed between the most global neuropsychological indicators (e.g, our z-score for global impairment and the H-R Index) after the time after the injury and between the two assessments were held constant. The results are presented in Table 7. After covarying for the two sources of time, there was no appreciable change in the strength of the correlation between sets of scores between the two periods of assessment. Covari- ance for chronological age also did not appreciably change either the magnitude of the differences or the strength of the association (Pearson $r$ ) between pairs of like measures.

Electroencephalographic measures, as well as the reports of types of medications and alterations in motivational variables (i.e., the ones reported in Tables 3-5 for the major population), revealed no statistically significant changes between the first and second assessments. The means or proportions were also comparable to the values for the major population reported in Table 1 for patients who were assessed between 1 and 2 years after the injury.

\subsection{Increment Changes during First Year after Injury}

The mean $\mathrm{z}$ scores for the various neuropsychological tests that demonstrated statistically significant (all Fs $=$ 1.54) differences $(p<0.01)$ for the 62 patients who were tested during different 0.1 year increments within 1 year of the brain trauma are shown in Table 8. Polynomial analysis indicated that only the linear terms were statistically significant. The $\mathrm{F}$ ratios for the weighted values (to accommodate disproportional numbers of patients per interval) are also shown. Patients tested between 0.3 years and 1.0 years after the injury displayed a significant linear increase in generalized deficits (mean z-score and the H-R Index) as well as deficits for visuospatial organization and memory, memory and localization of tactile shapes, and toe graphaesthesia. Neither the quadratic or cubic terms were statistically significant.

There were no statistically significant group differences for other components of the assessment battery (e.g., all of the measures shown in Table 1) such as the Category Test or the latency to complete the TPT for either the dominant or non-dominant hand. The only significant change in the MMPI scales occurred for the defensive scale which increased over time. There was no statistically significant linear term for the mean score for the numbers of complex partial epileptic-like experiences and phenomenology. There was no significant discrepancy with respect to age $(\mathrm{F}<1)$ or disconcordance for gender (chi-squared $<1.50$ ) between the temporal intervals.

There were two statistically significant changes in the electroencephalographic measures that may be relevant. The first involved the proportion of patients who displayed theta bursts within the prefrontal region (chisquared $=8.66$ ). These events emerged only in the groups that were assessed 0.6 years after the injury and involved $20 \%$ to $33 \%$ of the patients in each temporal increment. The second change involved the proportion of patients showing anomalies, such as odd spiky waves, bursts of theta patterns or vascular indicators that are strongly correlated with differential pulse height within 
Table 6. Test-retest scores for patients who were reassessed within 1.5 years after the first assessment. Paired t-test values for changes between the two test periods and the correlations (r) between the scores for the first and second assessment are also shown.

\begin{tabular}{|c|c|c|c|c|c|c|}
\hline \multirow{2}{*}{ Variable } & \multicolumn{2}{|c|}{ 1st Assess } & \multicolumn{2}{|c|}{ 2nd Assess } & \multirow{2}{*}{ Value } & \multirow{2}{*}{ corr } \\
\hline & $\mathbf{M}$ & SD & $\mathbf{M}$ & SD & & \\
\hline \multicolumn{7}{|c|}{ Standardized score } \\
\hline VIQ & 85.2 & 8 & 86.8 & 5.6 & 1.38 & 0.76 \\
\hline PIQ & 82.7 & 5.9 & 85.3 & 8.5 & 1.92 & 0.71 \\
\hline WMS (MQ) & 87.4 & 12.5 & 88.8 & 10.7 & 0.62 & 0.63 \\
\hline Achieve & 84.3 & 12.9 & 87.4 & 13 & 2.05 & 0.87 \\
\hline Z-impair & -3.1 & 1.1 & -2.7 & 1.1 & 1.59 & 0.68 \\
\hline \multicolumn{7}{|c|}{ Neuropsychological indicators (raw scores) } \\
\hline H-R Index & 0.7 & 0.2 & 0.6 & 0.2 & 1.21 & 0.72 \\
\hline Finger Tap R & 40.6 & 9.3 & 39.5 & 10.3 & 0.68 & 0.74 \\
\hline Finger Tap L & 33.8 & 11.3 & 31.3 & 12.2 & 1.44 & 0.77 \\
\hline Foot Tap R & 30.9 & 9.3 & 34.2 & 11.6 & 2.07 & 0.79 \\
\hline Foot Tap L & 24.9 & 8.6 & 25.3 & 10.4 & 0.25 & 0.81 \\
\hline Trails A & 68.6 & 26.7 & 60.6 & 25.1 & 1.48 & 0.56 \\
\hline Trails B & 131.5 & 79.7 & 119.1 & 72.1 & 0.86 & 0.67 \\
\hline Category & 79.4 & 20.8 & 65.3 & 28.9 & 2.06 & 0.28 \\
\hline Design F. & 15.2 & 11.8 & 16.4 & 8.6 & 0.45 & 0.44 \\
\hline CSAT & 114.3 & 53.5 & 86.3 & 53.6 & $3.01^{*}$ & 0.69 \\
\hline RO-copy & 27.9 & 4.5 & 28.6 & 3.3 & 0.55 & 0.28 \\
\hline RO-mem & 11.2 & 6.1 & 14.5 & 5.5 & $3.01^{*}$ & 0.76 \\
\hline TPT-D & 7.5 & 2.5 & 7 & 2.5 & 1 & 0.66 \\
\hline TPT-ND & 7.2 & 2.8 & 7.2 & 2.8 & 0.04 & 0.82 \\
\hline TPT-MEM & 5.6 & 1.5 & 6 & 1.7 & 0.84 & 0.48 \\
\hline TPT-LOC & 2.5 & 2.4 & 2.4 & 2.5 & 0.1 & 0.49 \\
\hline Speech S. & 22.2 & 4.6 & 22.5 & 4.3 & 0.31 & 0.59 \\
\hline Seashore & 23.1 & 3.1 & 123.8 & 3.2 & 1 & 0.5 \\
\hline Aud Clos & 14.3 & 5.5 & 16.4 & 5.4 & $2.40^{*}$ & 0.76 \\
\hline Sent Mem. & 15.4 & 3.5 & 15.4 & 3 & 0.08 & 0.61 \\
\hline Verbal Fl. & 25.1 & 10.8 & 27.9 & 8.7 & 1.23 & 0.47 \\
\hline Chicago WF & 26.9 & 13.2 & 28.7 & 17 & 0.84 & 0.88 \\
\hline Dich-LE & 31.6 & 5.5 & 32.2 & 5.6 & 0.33 & 0.16 \\
\hline Dich-RE & 34.2 & 12 & 32.5 & 13.2 & 0.94 & 0.86 \\
\hline Stroop CW & 34.4 & 8.1 & 35.5 & 9.3 & 0.72 & 0.74 \\
\hline Fing Agn $\mathrm{R}$ & 1 & 1.2 & 1.1 & 1.7 & 0.3 & 0.53 \\
\hline Fing Agn L & 1.6 & 1.8 & 1.9 & 1.7 & 0.88 & 0.61 \\
\hline Fing Graph R & 3.7 & 2.7 & 3.1 & 2.5 & 1.03 & 0.52 \\
\hline Fing Graph L & 3.4 & 3 & 2.7 & 2.5 & 1.13 & 0.51 \\
\hline Toe Agn L & 8.5 & 12.1 & 8.7 & 7.7 & 0.09 & 0.57 \\
\hline Toe Agn R & 6.6 & 3 & 7.5 & 3.3 & 1.06 & 0.36 \\
\hline Toe Graph R & 7.9 & 4.3 & 8 & 3.8 & 0.08 & 0.71 \\
\hline Toe Graph L & 7.5 & 3.8 & 8.2 & 3.9 & 1.26 & 0.85 \\
\hline CPES-signs & 69 & 61 & 52 & 45 & 1.9 & 0.91 \\
\hline
\end{tabular}

${ }^{*} \mathrm{p}<0.05,{ }^{* *} \mathrm{p}<0.01 ; \mathrm{r}>0.5, \mathrm{p}<0.01 ; \mathrm{r}>0.72, \mathrm{p}<0.001$. 
Table 7. Correlations between the first and second assessment scores for the major indicators of brain impairment after the time since the injury or time between the two assessments was held constant.

\begin{tabular}{cccc}
\hline Variable assess & zero-order & Time since injury & Time since 1st \\
\hline H-R index & 0.72 & 0.73 & 0.72 \\
z-global & 0.68 & 0.7 & 0.69 \\
Dichotic-R ear & 0.86 & 0.88 & 0.85 \\
Performance IQ & 0.71 & 0.75 & 0.73 \\
\hline
\end{tabular}

all values $\mathrm{p}<0.001$.

Table 8. Mean z-scores for measurements that displayed a statistically significant $(p<0.01)$ weighted linear term for increased impairment during the first year after the brain injury ( $n$ = numbers of patients per time increment).

\begin{tabular}{|c|c|c|c|c|c|c|c|c|c|}
\hline \multirow{2}{*}{ Variable } & \multicolumn{9}{|c|}{ Time (in decimals of year) since injury } \\
\hline & 0.3 & 0.4 & 0.5 & 0.6 & 0.7 & 0.8 & 0.9 & 1 & Linear \\
\hline $\mathbf{n}$ & 8 & 4 & 15 & 5 & 8 & 6 & 3 & 13 & Trend \\
\hline H-RIndex & 0.36 & 0.3 & 0.53 & 0.64 & 0.49 & 0.73 & 0.83 & 0.66 & $8.84^{*}$ \\
\hline z-mean & -0.7 & -0.6 & -1.4 & -1.6 & -1 & -2.1 & -3 & -2 & $7.15^{* *}$ \\
\hline Foot-R & -0.2 & 0 & -0.4 & -0.8 & -0.5 & -1.3 & -0.8 & -1.1 & $7.41^{*}$ \\
\hline Foot-L & -0.4 & -0.4 & -0.7 & 0.7 & -0.3 & -1.3 & -0.9 & -1.4 & $7.75^{*}$ \\
\hline R-O Copy & -0.7 & -0.2 & -0.7 & -0.6 & -0.8 & -2 & -1.9 & -1.7 & $8.29^{*}$ \\
\hline R-O Mem & -1.3 & 0.1 & -1.2 & 0.7 & -0.4 & -2.4 & -2.1 & -2.1 & $9.83^{*}$ \\
\hline TPT-Mem & -1.2 & -1.3 & -3.1 & -2.3 & -1.8 & -2.5 & -4 & -3.1 & $7.31^{*}$ \\
\hline TPT-Loc & -0.6 & -1.6 & -2.8 & -2.4 & -2.5 & -3 & -3.9 & -3.1 & $10.15^{*}$ \\
\hline Stroop WC & -0.4 & -0.5 & -0.8 & -0.7 & -1.3 & -1.3 & -2 & -1.4 & $10.17^{*}$ \\
\hline Toe Gr (d) & -0.4 & -0.3 & -1.5 & -1.7 & -1 & -1.7 & -1.9 & -2 & $5.92^{*}$ \\
\hline MMPI (K) & 44 & 38 & 48 & 48 & 52 & 54 & 42 & 56 & $11.86^{*}$ \\
\hline
\end{tabular}

${ }^{*} \mathrm{p}<0.01 ;{ }^{* *} \mathrm{p}<0.001$.

the right frontooccipital montage. This was due to the transient appearance of these anomalies within $25 \%$ to $40 \%$ of the patients who were assessed 0.4 through 0.8 years after the injury but not before or after this increment.

Because of the potential confound from the consequences of the induction of consciousness from the injury, the 81 patients within the first analysis whose records contained this verified information were compared according to the report of no unconsciousness $(n=37)$, unconsciousness up to $5 \mathrm{hr}(\mathrm{n}=23)$ and unconscious for more than $5 \mathrm{hr}(\mathrm{n}=21)$. The results are shown in Table 9 . Three patterns were evident. First, patients who reported no unconsciousness displayed deficits that were more similar to patients who had experienced protracted unconsciousness. Second, the occurrence of theta bursts over the temporal and frontal regions was clearly evident for patients who had been rendered unconscious. Third, patients who experienced no "unconsciousness" were still reporting a significant increase in complex partial epileptic-like signs compared to both the groups who had been rendered unconscious.

\section{Discussion}

The results of this study indicated that there were no statistically significant differences between about 0.5 years and about 4 years after a brain trauma within the general domains of cognition, memory, neuropsychological proficiency and efficiency or personality. The levels of intelligence, global memory capacity and educational achievement were all within the average range for the groups of patients that were assessed within 1 year, between 1 and 2 years, between 2 and 3 years or more than 3 years after the injury. Only the average score for the group assessed within one year after the injury would have met the cut-off criterion (Impairment Index $>0.4$ ) for brain impairment according to the traditional definition. However according to ordinal ranks of neuropsychological impairment $[15,16]$, all of the groups tested at different times after the injury would have displayed, on average, mild to moderate neuropsychological deficits that did not 
change over time.

A comparable magnitude of impairment, that also did not differ significantly between the post-injury assessment times, was noted for our global impairment index which was derived from the average of 31 standardized scores from a variety of traditional and contemporary neuropsychological procedures [3]. When traditional criteria of brain impairment were applied, i.e., a significantly impaired neuropsychological function relative to cognition, there was also no significant disconcordance in incidence of these patients across the four assessment periods. About one-quarter of the patients displayed a magnitude of neuropsychological impairment that would be sufficient, particularly if they were professionals such as lawyers, physicians, or professors, to interfere with cognitively complex, frequently changing, vocational demands.

These results are consistent with the hypothesis that a brain trauma sufficient to evoke symptoms that interfere with adaptation and ultimately result in referral for assessment and treatment could produce deficits that do not "recover" or improve over time. Instead, the patient adapts to the changes in potential. Because this adaptation usually involves the restriction of activities or a reduction in vocational demands, the person may appear to "recover" $[3,16,17]$. However these deficits would become conspicuous to the patient and the family when the patient attempts to acquire new concepts within novel vocational contexts.

The general class of medications that were administered to this population of patients did not differ between the groups that were assessed at different times after the injury. Over one-third of these patients had been consuming analgesics. The incidence of posttraumatic seizures included $16 \%$ of the population which indicates that electrical lability may be more prominent than suspected and is not related to the severity of the neuropsychological impairment or the reported occurrence of unconsciousness. In fact the patients who did not report unconsciousness exhibited marked increases in symptoms and experiences that are typical of complex partial epileptic patients (Table 9). It may be relevant that female rats that were stunned by an impact of 200 gm dropped from 0.9 meters displayed significantly more severe impairments during the acquisition of a Morris Water Maze than rats that had been rendered unconscious by this process who did not differ from controls [6-8].

Table 9. Means and standard deviations for primary measures as a function of duration (in hr) of unconsciousness.

\begin{tabular}{|c|c|c|c|c|c|c|}
\hline \multirow{3}{*}{ Variable } & \multicolumn{2}{|c|}{ No unconsciousness } & \multicolumn{2}{|c|}{ Minimal-5 hr } & \multicolumn{2}{|c|}{$>5 \mathrm{hr}$} \\
\hline & \multicolumn{2}{|c|}{$\mathbf{n}=\mathbf{3 7}$} & \multicolumn{2}{|c|}{$n=23$} & \multicolumn{2}{|c|}{$\mathbf{n}=\mathbf{2 1}$} \\
\hline & M & SD & $\mathbf{M}$ & SD & $\mathbf{M}$ & SD \\
\hline Verbal intel & 86 & $10 \mathrm{a}$ & 94 & $10 \mathrm{~b}$ & 89 & 10 \\
\hline Perform intel & 90 & 13 & 100 & $15 \mathrm{a}$ & 88 & $12 \mathrm{~b}$ \\
\hline H-R index & 0.48 & $0.27 \mathrm{a}$ & 0.34 & $0.32 \mathrm{~b}$ & 0.5 & $0.25 \mathrm{a}$ \\
\hline z-impairment & -1.6 & $1.4 \mathrm{a}$ & -1 & $1.2 \mathrm{~b}$ & -1.4 & 1 \\
\hline Trails A & 37 & $17 \mathrm{a}$ & 38 & $19 \mathrm{a}$ & 52 & $28 \mathrm{~b}$ \\
\hline Rey-Copy & 28 & $4 \mathrm{a}$ & 31 & $3 b$ & 29 & 3 \\
\hline TPT-D & 6.5 & $2.7 \mathrm{a}$ & 4.6 & $2.2 \mathrm{~b}$ & 7 & $2.6 \mathrm{a}$ \\
\hline TPT-ND & 5.2 & 2.6 & 4 & $2.3 \mathrm{a}$ & 6.5 & $3.1 \mathrm{~b}$ \\
\hline Temporal alpha & 5.6 & $10.7 \mathrm{a}$ & 17.8 & $24.0 \mathrm{~b}$ & 9.1 & $13.4 \mathrm{a}$ \\
\hline \multirow[t]{2}{*}{ Roberts' CPES } & 85 & $37 \mathrm{a}$ & 57 & $35 \mathrm{c}$ & 48 & $37 \mathrm{c}$ \\
\hline & \multicolumn{2}{|c|}{$\%$} & \multicolumn{2}{|c|}{$\%$} & \multicolumn{2}{|c|}{$\%$} \\
\hline Frontal theta & \multicolumn{2}{|c|}{$2 a$} & \multicolumn{2}{|c|}{$22 b$} & \multicolumn{2}{|c|}{$19 \mathrm{~b}$} \\
\hline Temporal theta & \multicolumn{2}{|c|}{$0 \mathrm{a}$} & \multicolumn{2}{|c|}{$17 \mathrm{~b}$} & \multicolumn{2}{|c|}{$14 \mathrm{~b}$} \\
\hline Occipital theta & \multicolumn{2}{|c|}{2} & \multicolumn{2}{|c|}{1} & \multicolumn{2}{|c|}{4} \\
\hline Occipital pulse delay & \multicolumn{2}{|c|}{$27 \mathrm{a}$} & \multicolumn{2}{|c|}{$4 \mathrm{~b}$} & \multicolumn{2}{|c|}{9} \\
\hline
\end{tabular}

a vs b; $p<0.05 ;$ a vs c; $p<0.01$. 
The results of our electroencephalographic analysis indicated that anomalous events, that were conspicuously evident by qualitative analysis, were significantly more prominent for the group of patients who were assessed within one year of the trauma. The importance of this first year was reiterated by the quantitative measures that showed a significant increase in the proportion of alpha rhythms (per min) over the occipital region but not over the temporal and frontal regions after the first year of injury. The capacity to generate alpha rhythms over the posterior regions has, in general, been associated with relaxation and decreased anxiety or non-specific arousal. Although there were disproportionately more trains of epileptiform activity in the records of patients who had sustained the brain trauma more than 3 years previously compared to the other groups, a "delayed" exacerbation of electrical anomalies may not be the only explanation. It is possible that these patients may have been referred because of intractable difficulties coupled to the frequency of seizure activity which could be easily unmasked during the relaxing period following a full day of cognitive testing.

However the major source of variance within psychological and neuropsychological measurements is individual differences. The counterargument to the failure to discern significant change in neuropsychological tests and the persistent impairment over time would be that any small changes were masked by these differences. In addition, translation of raw scores into standardized values ignores the range of 4 standard deviations that constitutes "normality" $[1,4]$. Failure to accommodate this concept may help explain statements such as "mild head injury in general is not associated with long-term persistent neuropsychological impairments" [18].

For example, if a person within a professional vocation displayed a premorbid intellectual level that was +1.5 standard deviations above the average but after a diffuse injury displays a full scale score that is -1.0 , the person would still be considered "average" even though, technically, a significant decrement of 2.5 standard deviations had occurred. Comparably impaired neuropsychological scores, referenced to this postincident level of intelligence, would not be discrepant. Consequently the conclusion such as "no discrepancy, hence no brain impairment" might be considered even though this global decrement has clearly interfered with the professional's capacity to adapt to the vocational setting.

The test-retest scores of 20 patients who were tested first, on average, between 0.5 and 1.5 years after the injury and then 1.0 to 1.5 years later, in general verified the persistence of the neuropsychological deficits in context of average intelligence, memory and educational achievement. This group of patients by definition was impaired [as defined by standardized (global z score) im- pairments that were moderate $(>-2.0)$ to moderately severely $(>-3.0)$ impaired and significantly below their intellectual/general memory capacity] during the first assessment and was still impaired during the second assessment. The test-retest correlations on average accommodated more than $50 \%$ of the variance $(r>0.70)$. We suspect that the magnitudes of the test-retest correlations would have been even higher but were attenuated because of the restricted range of the scores due to the level of the global impairments.

The only scores for tests that displayed improvement between the two periods of assessment included the Conditioned Spatial Association Test, Auditory Closure, the memory component of the Rey-Ostereith Figure and the psychasthenia $(\mathrm{Pt})$ component of the MMPI. The most parsimonious source of shared variance between these four measures would involve the right orbitofrontal region or related areas of the right anterior temporal region. The decreased generalized vigilance (anxiety) would be expected as right anterior hemispheric function improved and exhibited the normal inhibition over right (subcortical) temporal and limbic regions. Additional support for the specific changes within the right anterior hemisphere was indicated by the appearance of electrical anomalies within the right prefrontal region only during the first year after the injury in a separate group of patients.

Whether or not the improvements in the scores for the tests that have been inferred to reflect right prefrontal function is indicative of a return of metabolic activity following a period of hypometabolic function or suggests a preferential influence of transfer from the first assessment cannot be differentiated. The size of the effects for these changes in the three neuropsychological tests explained approximately $23 \%$ to $34 \%$ of the variance. Normally a change or treatment explains or accommodates about $40 \%$ to $50 \%$ of the variance in order to also be correlated with clinically discernable changes within the individual patient. However despite these specific changes, these patients still displayed neuropsychological deficits. Because the deficits were not confounded by time since the injury, time between the first and second assessment or with age (as indicated by partial correlation analysis) and were more than expected by the standardized levels of intelligence and educational achievement, these patients still displayed mild to moderate brain impairment.

One traditional premise within rehabilitation psychology and clinical neuropsychology is that the patient "improves" over time and that within about 6 months after the injury there is no significant difference between patients who sustain mild brain injuries and age-matched controls. This comment is derived from asymptote theories for which there is little valid or empirical evidence. The results of this study indicate that patients who have sustained what would typically be labelled as "mild to 
moderate" brain injuries display a progressive deterioration (Table 9) that is linear between 0.3 years to 1.0 year after the trauma. The exacerbation of these deficits is even accompanied by the conspicuous increased proportion of theta rhythms within screening EEGs as well as the appearance of electrical anomalies over the right prefrontal regions.

Standardized (z) scores (Table 9) indicate that whereas patients who were assessed within 0.3 to 0.4 years after the injury exhibited scores within the average range $(\mathrm{z}$ scores between -1.00 to +1.00 ), the deficits increased to worse than -1.00 and even abnormal $(-2.0$ to -4.0$)$ levels for groups that were assessed between 0.9 to 1.0 year after the injury. This linear effect was not confounded by the age, educational achievement, or intellectual level of the patients. The similarities of the effects for two subsamples, whose referral policies had involved either assessment because of complications or more or less random assignment for the assessment after the time since the injury, strongly suggest that this phenomenon was not an artifact of the magnitude of the initial injury, i.e., the more recalcitrant patients are only referred later after initial rehabilitative attempts failed.

During the last decade at least two groups of clinical neuroscientists $[13,14,19]$ have been pursuing the hypothesis that a continuum of temporal lobe lability or sensitivity exits within the human population. Following brain trauma, this lability may progress into an Epileptic Spectrum Disorder that is similar to the partial complex epilepsy with foci within the limbic system except the patient does not exhibit neurologically defined epileptic seizures. The results of this study indicate that a significant proportion of patients who sustained head injuries also reported experiences that are typical of epileptic patients diagnosed with partial complex seizures without generalization to tonic-clonic convulsions. The scores for the Roberts' Inventory of complex partial epileptic-like signs (Roberts et al., 1990) were more than 3 standard deviations above the average population. The magnitudes of these scores were not significantly different between the groups assessed at various times after the injury or within the same group who was assessed twice. If these persistently elevated scores reflected increased "neuroelectrical noise", as suggested by Dick Roberts et al. [14] and reiterated by Shaw [20] then the maintained problems reported by these patients for "concentration", remembering new information, and, responding to context, which are classic indicators of dysfunctional hippocampal activity, might be explained.

The comparisons of patients as a function of the duration of unconsciousness may have revealed a major source of this large coefficient of variation (in addition to the expected correlates of location of the brain trauma). Patients who did not report unconsciousness (but a pe- riod of unreality or confusion) reported marked elevations in the daily and weekly incidence of experiences similar to those reported by complex partial epileptic patients. On the other hand, patients who sustained unconsciousness displayed more traditional electroencephalographic anomalies, such as theta bursts over the temporal and frontal regions. This pattern is reminiscent of the "forced normalization" phenomenon which involves a reciprocal relationship between limbic (mesiobasal temporal) phenomena and cortical (EEG) anomalies. "Normal" EEG activity is associated with cognitive abnormalities while abnormal EEG activity is associated with apparently normal cognitive functions.

If this concept is generalizable to this population, then the hypothesis that a significant proportion of the patients who "did not experience unconsciousness" experience subclinical electrical anomalies that simulate complex partial seizures should be pursued. The behavioral correlates of these changes would be manifested primarily within the domain of "psychiatric" or "psychological" indicators and could be misconstrued with respect to both aetiology and treatment. There is now evidence, within the animal literature, that intermittent but insidious seizure disorders might encourage neuronal dysfunction and death over periods of months to years until the "electrically labile" neurons die or are controlled by the appropriate medication [21].

Wudu and Persinger [6-8] found that rats who sustained a mechanical impact to their skulls exhibited apoptoticlike shrinkage of some cortical neurons within specific "zones" beneath the impact site about one month after the injury. This conspicuous cytomorphic change was evident in rats that had been only mildly stunned (disrupted behavior of less than $10 \mathrm{sec}$ ) or rendered unconscious for several minutes by the blow. There was no evidence of oedema or local reactions. Such changes, even if the required resolution (about 10 micrometers) were currently possible, would not have been easily discerned by the methodology employed by either CT (Computerized Tomography) or MRI (Magnetic Resonance Imaging) to infer brain structure.

\section{Conclusion}

The results of this study indicate that the traditional assumptions concerning "improvement" after mild to moderate brain injury may not be valid when objective, norm-referenced measures rather than clinical impressions or reports by patients and family are employed as criteria. There may be non-linearities in the display of deficits such that they are particularly exacerbated between about 6 months to one year after an injury that is labelled as "only a concussion" (Shaw, 2002) or mild brain injury at the time of the admission to emergency settings. The effects within this population may even be 
evident by screening electroencephalographic indicators. This conspicuous observation emphasizes the potential utility of this tool for clinical neuropsychologists. It would be employed to verify the validity of the results of the neuropsychological tests and would not replace the full assessment by the neurologist.

The clinical implication of these results is that patients who have sustained a "mild brain injury" should be monitored for longer periods than previously considered. A "return to work" within a few weeks should not be used necessarily as "proof" that the person has "recovered" and hence subsequent "problems" are indicative of neurosis, depression or "learning to be impaired". We have seen, during multiple occasions, professionals who return to work within two or three weeks after a mild head injury only to deteriorate so significantly that "sick leave" is required for several months about 6 months to one year after the injury. If during this period there is a marked disequilibrium within the anterior right prefrontal (and orbital frontal) region which disinhibits the right temporal and right parahippocampal regions, then the enhanced anxiety, spatial confusion and disturbances in the sense of self would be expected. The could explain the frequent emergence of complex partial epileptic-like experiences following "mild to moderate" closed head injuries [22].

\section{Acknowledgements}

Thanks to Professor Blake T. Dotta for technical assistance.

\section{REFERENCES}

[1] M. A. Persinger, "Neuropsychologica Principia Brevita: An Application to Traumatic (Acquired) Brain Injury," Psychological Reports, Vol. 77, No. 3, 1995, pp. 707-724. doi:10.2466/pr0.1995.77.3.707

[2] M. A. Persinger, "Personality Changes Following Brain Injury as a Grief Response to Loss of the Sense of Self: Phenomenological Themes and Indices of Local Lability and Neurocognitive Structuring as Psychoptherapy," Psychological Reports, Vol. 72, No. 3, 1993, pp. 1059-1068. doi:10.2466/pr0.1993.72.3c.1059

[3] M. A. Persinger, "Clinical Neurological Indicators Are Only Moderately Correlated with Quantitative Neuropsychological Test Scores in Patients Who Display Mild-Moderate Brain Impairment Following Closed Head Injuries," Perceptual and Motor Skills, Vol. 81, No. 3, 1995, pp. 1283-1292. doi:10.2466/pms.1995.81.3f.1283

[4] R. S. Parker, "Traumatic Brain Injury and Neuropsychological Impairment,” Springer-Verlag, New York, 1990. doi:10.1007/978-1-4612-3398-5

[5] R. Schoenhuber and M. Gentilini, "Neuropsychological Assessment of Mild Head Injury," In: H. S. Levin, H. M. Eisenberg and A. L. Benton, Eds., Mild Head Injury, Oxford University Press, New York, 1989, pp. 101-121.

[6] W. E. Lado and M. A. Persinger, "Mechanical Impacts to the Skulls of Rats Produce Specific Deficits in Maze Performance and Weight Loss: Evidence of Apoptosis of Cortical Neurons and Implications for Clinical Neuropsychology," Perceptual and Motor Skills, Vol. 97, 2003, pp. 115-127.

[7] W. E. Lado and M. A. Persinger, "Increased Conditioned Immobility and Weight Loss in Rats Following Mechanical Impacts to the Skull that Do Not Produce Loss of Consciousness," Central European Journal of Biology, Vol. 3, No. 4, 2008, pp. 322-430. doi:10.2478/s11535-008-0041-6

[8] W. E. Lado and M. A. Persinger, "Spatial Memory Deficits and Their Correlations with Clusters of Shrunken Neuronal Soma in the Cortices and Limbic System Following a "Mild" Mechanical Impact to the Dorsal Skull in Female Rats," Journal of Behavioral and Brain Science, Vol. 2, 2012, pp. 333-342. doi:10.4236/jbbs.2012.23038

[9] H. S. Levin, "Neurobehavioral Sequelae to Closed Head Injury,” In: R. P. Cooper, Ed., Head Injury, William \& Wilkins, Baltimore, 1993, pp. 525-551.

[10] M. A. Persinger and P. M. Richards, "Foot Agility and Toe Gnosis/Graphaesthesia as Potential Indicators of Integrity of the Medial Surface: Normative Data and Specific Pop pulations," Perceptual and Motor Skills, Vol. 80, No. 3, 1995, pp. 1101-1024. doi:10.2466/pms.1995.80.3.1011

[11] A. Salmoni, P. M. Richards and M. A. Persinger, "Absence of Frontal Lobe Dysfunction Indicators in Healthy Elderly Subjects: Comparisons with Verified Frontal Lobe Damage," Developmental Neuropsychology, Vol. 12, No. 2, 1996, pp. 201-206. doi:10.1080/87565649609540646

[12] K. R. Vincent, I. M. Catillo, R. I. Hansen, J. A. Zapata, H. J. Stuart, C. K. Cohn and G. J. O'Shanick, "MMPI-168 Codebook,” Norwood (N.J.), Ablex, 1984.

[13] R. J. Roberts, L. L.Gorman, G. P. Lee, M. E. Hines, E. D. Richardson, T. A. Riggle and N. R. Varney, "The Epileptic Spectrum Disorder," Epilepsy Research, Vol. 13, No. 2, 1992, pp. 167-177. doi:10.1016/0920-1211(92)90073-3

[14] R. J. Roberts, N. R. Varney, J. R. Hulbert, J. S. Paulsen, J. S. Richardson, J. A. Springer, J. S. Shepherd, C. M. Swan, J. A. Legrand, J. H. Harvey, M. A. Struchen and M. E. Hines, "The Neuropathology of Everyday Life: The Frequency of Partial Seizure Symptoms Among Normals," Neuropsychology, Vol. 5, 1990, pp. 75-85.

[15] E. Neidermeyer and F. L. Da Silva, "Electroencephalography: Basic Principles, Clinical Applications and Related Fields," Ubran and Schartzenberg, Baltimore, 1987.

[16] M. A. Persinger, "Discrepancies between Standardized Measures of Cognitive Level and Halstead-Reitan Impairment Indices as Inferences of Brain Damage Following Head Injuries," Perceptual and Motor Skills, Vol. 89, No. 3, 1999, pp. 1210-1216. doi:10.2466/pms.1999.88.3c.1210

[17] M. A. Persinger, "Sense of Presence and Suicidal Ideation Following Brain Injury: Indications of Right Hemispheric Intrusions from Neuropsychological Profiles," Psychological Reports, Vol. 75, No. 3, 1995, pp. 1059-1070. doi:10.2466/pr0.1994.75.3.1059

[18] S. S. Dikmen, J. E. Machamer, H. R Winn and N. R. Tem- 
kin, "Neuropsychological Outcome One-Year Post Head Injury," Neuropsychology, Vol. 9, No. 1, 1995, pp. 80-90. doi:10.1037/0894-4105.9.1.80

[19] M. A. Persinger and K. Makarec, "Temporal Lobe Epileptic Signs as a Continuum from Normals to Complex Partial Epileptics: Normative Data and Special Populations," Journal of Clinical Psychology, Vol. 40, No. 1, 1993, pp. 33-45.

doi:10.1002/1097-4679(199301)49:1<33::AID-JCLP2270 490106>3.0.CO;2-H

[20] N. A. Shaw, "The Neurophysiology of Concussion," Progress in Neurobiology, Vol. 67, No. 4, 2002, pp. 281-334. doi:10.1016/S0301-0082(02)00018-7
[21] G. F. Lafreniere, O. Peredery and M. A. Persinger, "Progressive Accumulation of Large Aggregates of Calcium Containing Polysaccharides and Basophilic Debris within Specific Thalamic Nuclei after Lithium/Pilocarpine-Induced Seizures," Brain Research Bulletin, Vol. 28, No. 5, 1992, pp. 825-830. doi:10.1016/0361-9230(92)90268-3

[22] R. Gorham and M. A.Persinger, "Emergence of Complex Partial Epilepsy-Like Experiences Following Closed Head Injuries: Personality Variables and Neuropsychological Profiles," Epilepsy and Behavior, Vol. 23, No. 2, 2012, pp. 152-158. doi:10.1016/j.yebeh.2011.11.010 